\title{
Development of Finite Element Model for Analysis of Rolling Contact Fatigue Cracks in Wheel/Rail Systems
}

\author{
Makoto AKAMA, Ph.D. \\ Senior Researcher, Vehicle Strength, \\ Vehicle Structure Technology Division.
}

\begin{abstract}
The problem of rolling contact fatigue cracks that occur on wheel and rail surfaces is one of the most significant in the railway industry. This paper introduces a finite element method (FEM) model that has been developed for the analysis of such cracks. The model consists of FEM analysis that takes into account the local material response generated by the contact load between wheel and rail. Both the wheel and the rail are modeled using an FEM mesh, and the wheel is loaded and rotated in the rolling direction on the rail. Combined isotropic and kinematic hardening law was used to model the decaying hardening rate that is important in rolling contact situations. Stress and strain values obtained through FEM analysis were combined with the multi-axial fatigue life model and the critical plane approach to predict the onset of fatigue cracks in the railhead. The calculated results were compared to the data obtained from investigation in the field, and discussions were made.
\end{abstract}

Keywords: finite element method, rolling contact fatigue, multi-axial fatigue, wheel, rail

\section{Introduction}

The problem of rolling contact fatigue (RCF) cracks that occur on wheel and rail surfaces is one of the most significant in the railway industry. In rails, the two types of surface-initiated cracks identified are head checks and squats. Head checks form primarily along the gauge corner of the high rail on curves where there is contact between wheel and rail. These cracks are shallow-angled and very small. The direction of head checks follows the direction of the deformed material microstructure generated by traction forces. Squats, on the other hand, are cracks that occur on the crown of the railhead. They start as tiny cracks and extend down into the railhead at a shallow angle to the upper surface in the direction of traffic. Once they reach a certain length, they start to form branch cracks, which will lead to catastrophic failure if left to grow. These cracks occur frequently on tracks that carry high-speed trains and those that have a high annual tonnage, and represent a particularly serious problem for railway companies all over the world. Rail companies have for some time ground the surface of tracks to remove RCF cracks and corrugation. It has been suggested that grinding away more of the rail in areas susceptible to squats may eliminate such cracks before they reach any great depth. The problems associated with this method involve the amount of rail removed and the interval between removals.

In the area of head check crack initiation, extensive finite element models have been developed by Ringsberg et al. ${ }^{12)}$. These models include cycle-by-cycle analysis of ratchetting strain accumulation, low-cycle fatigue and the critical plane approach. A search algorithm was used to find the most damaging plane by using the stress and strain tensors obtained from finite element analysis. The research for squats ${ }^{3)}{ }^{4)}$ seems to concentrate on co-pla- nar growth, and studies on the initiation of squat cracks is lacking.

In this paper, therefore, numerical modeling for squat-crack initiation will be developed with reference to the work carried out by Ringsberg et al. The finite element method (FEM) is utilized to predict the position and direction of cracks and the fatigue life. The proposed model uses a combined isotropic and kinematic hardening law that is necessary to simulate low-cycle fatigue behavior with a decaying hardening rate. The results of FEM analysis are then utilized to predict crack initiation using one of the criteria corresponding to the material responses to cyclic loadings.

\section{Finite Element Modeling}

This section outlines the basic knowledge of fatigue crack initiation, material responses to cyclic loading, models for fatigue crack initiation and simulation procedures in detail.

\subsection{Crack Initiation}

The life of a fatigue crack is normally divided into three phases covering crack initiation and growth. These are: Stage I - shear stress-driven initiation at the surface, Stage II - transient crack growth behavior, and Stage III - subsequent tensile- and shear-driven crack growth. Cracks start at rolling contact surfaces as a result of the accumulation of shear deformation from repeated rollingsliding contact loading. In this research, initiation may be considered to have occurred when stage I is complete. In railway tracks, this corresponds to crack sizes of about $0.5 \mathrm{~mm}$. 


\subsection{Shakedown ${ }^{5)}$}

Ductile metals are often subjected to cyclic loads in such a way that the early cycles lead to a build-up of residual stress. This stress can be of such a magnitude that a steady state is attained after load reversals where a closed cycle of entirely elastic reversed deformation is promoted. In this situation, there is no net accumulation of plastic strain in subsequent cycles, and the system is said to have undergone shakedown. Elastic shakedown occurs when the development of residual stress results in a steady state in which cyclic deformation is purely elastic. On the other hand, plastic shakedown refers to a state of deformation where a closed cycle of alternating plasticity occurs without any accumulation of plastic strain. If the limit of shakedown is exceeded, plastic strain continues to accumulate during each cycle in a phenomenon known as ratchetting. The material response in the wheel/rail contact region can be any of these types, and is presented in Fig. $1^{6)}$. The fatigue crack initiation criteria differ according to the type of material response. Examples of this are shown in the following section.

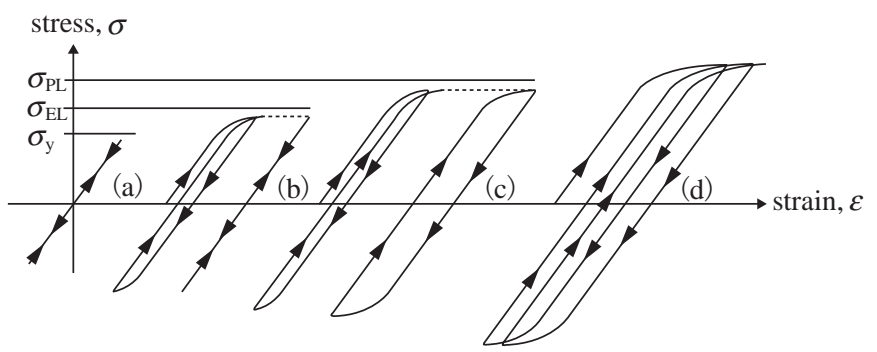

Fig. 1 Response of a material to cyclic loading: (a) purely elastic, (b) elastic shakedown, (c) plastic shakedown, (d) ratchetting ${ }^{6}$.

\subsection{Fatigue Crack Initiation Criteria}

(a) Elastic Shakedown

Goodmann and Haigh diagrams appear useful when predicting life under simple tensile or torsional loading with any mean value for high-cycle fatigue such as elastic shakedown material response. In RCF, however, the material near the surface experiences a non-proportional cycle of tension followed by shearing and then compression. This load gives rise to an out-of-phase rotation of principal stress and strain over time that makes it difficult to predict the position and direction of crack initiation. Some criteria, which are multi-axial extensions of Goodmann and Haigh's relation, are applicable. The uniaxial stress amplitude is replaced by a measurement of the amplitude of the equivalent von Mises or Tresca stresses. An example is the Sines criterion ${ }^{7)}$, which can be written as:

$$
\begin{aligned}
\frac{1}{2}\left\{\left(\Delta \sigma_{1}-\Delta \sigma_{2}\right)^{2}+\left(\Delta \sigma_{2}-\Delta \sigma_{3}\right)^{2}\right. & \left.+\left(\Delta \sigma_{3}-\Delta \sigma_{1}\right)^{2}\right\}^{\frac{1}{2}} \\
& +A\left(\bar{\sigma}_{1}+\bar{\sigma}_{2}+\bar{\sigma}_{3}\right)=C
\end{aligned}
$$

where $\Delta \sigma_{1}, \Delta \sigma_{2}$ and $\Delta \sigma_{3}$ are amplitudes of the principal stresses, $\bar{\sigma}_{1}, \bar{\sigma}_{2}$ snd $\bar{\sigma}_{3}$ are mean values over a cycle of the principal stresses, and $A$ and $C$ are material constants. The second term in equation (1) is the mean value of hydrostatic tension. The Crossland criterion ${ }^{8)}$ is written in a similar form, but this time the value stands for the maximum hydrostatic tension in the cycle.

Dang Van et al. ${ }^{9)}$ present a multi-axial fatigue limit criterion which suggests that initiation occurs if the combination of the shear stress amplitude value $\tau_{\mathrm{a}}(t)$ on a shear plane and the value of hydrostatic stress $\sigma_{h}(t)=\left(\sigma_{1}(t)+\sigma_{2}(t)+\sigma_{3}(t)\right) / 3$ at the material point under consideration fulfill one or both of the following inequalities during some parts $t_{1}<t<t_{2}$ of a full stress cycle:

$$
\tau_{a}(t)+a_{D V} \sigma_{h}(t)>\tau_{e} \text { or } \tau_{a}(t)-a_{D V} \sigma_{h}(t)<-\tau_{e}
$$

where $\tau_{e}$ is the positive fatigue limit of the material in pure shear and $a_{D V}$ is a material parameter representing the influence of hydrostatic stress.

(b) Plastic Shakedown

Smith and co-authors ${ }^{10)}$ proposed a simple form of SWT damage parameter (described as a product of stress and strain) for determining fatigue life under uni-axial loading in the case of low-cycle fatigue, i.e. plastic shakedown material response. The SWT parameter was used after the following modification in multi-axial proportional and non-proportional loading for materials with cracks according to mode I. The modification involves considering only stress and strain that occurs in the critical plane. The most popular form of SWT parameter in the critical plane is that proposed by Socie ${ }^{11)}$ in the plane of the maximum normal strain range $\Delta \varepsilon_{1}$

$$
\sigma_{n, \max } \frac{\Delta \varepsilon_{1}}{2}=\frac{\sigma_{f}^{\prime 2}}{E}\left(2 N_{f}\right)^{2 b}+\sigma_{f}^{\prime} \varepsilon_{f}^{\prime}\left(2 N_{f}\right)^{b+c}
$$

where $\sigma_{n \text {,max }}$ is the maximum normal stress in the plane with normal $n, \sigma_{f}^{\prime}$ and $\varepsilon_{f}^{\prime}$ represent the fatigue strength coefficient and fatigue ductility coefficient respectively, $E$ is Young's modulus, $b$ and $c$ are the fatigue strength exponent and the fatigue ductility exponent, and $N_{f}$ is the number of cycles to failure.

Rolovic and Tipton ${ }^{12)}$ proposed a criterion for multiaxial cyclic proportional and non-proportional fatigue loading including the mean value of normal stress. The specific form was proposed as

$$
\left(\tau_{n s, a}+0.3 \sigma_{n}\right) \gamma_{n s, a}+\sigma_{n, \max } \varepsilon_{n, a}=\frac{\sigma_{f}^{\prime}}{E}\left(2 N_{f}\right)^{2 b}+\sigma_{f}^{\prime} \varepsilon_{f}^{\prime}\left(2 N_{f}\right)^{b+c}
$$

where $\tau_{n s, a}$ is the shear stress amplitude in direction $s$ on the plane with normal $n, \sigma_{n}$ is the normal stress on the plane, $\sigma_{n, \max }$ is the maximum value of $\sigma_{n}$ over time and $\varepsilon_{n, a}$ is the amplitude of normal strain on the plane. The critical plane is determined as the one with the highest calculated damage level.

Chen et al. ${ }^{13)}$ proposed two criteria; the first one for materials characterized by mode I cracks, and the second for materials characterized by the mode II type. Both criteria assume the influence of normal and shear stress and strain in the critical plane on fatigue life. For materials characterized by mode I cracks, the critical plane is the plane of the maximum normal strain range $\Delta \varepsilon_{n}$

$$
\Delta \varepsilon_{n} \Delta \sigma_{n}+\Delta \gamma_{n s} \Delta \tau_{n s}=4 \frac{\sigma_{f}^{\prime 2}}{E}\left(2 N_{f}\right)^{2 b}+4 \sigma_{f}^{\prime} \varepsilon_{f}^{\prime}\left(2 N_{f}\right)^{b+c}
$$

where $\Delta \sigma_{n}$ is the normal stress range, $\Delta \gamma_{n s}$ is the maximum shear strain range and $\Delta \tau_{n s}$ is the maximum shear 
stress range. For materials with mode II cracks, the critical plane is the plane of the maximum shear strain range $\Delta \gamma_{n s}$

$$
\Delta \varepsilon_{n} \Delta \sigma_{n}+\Delta \gamma_{n s} \Delta \tau_{n s}=4 \frac{\tau_{f}^{\prime 2}}{E}\left(2 N_{f}\right)^{2 b_{0}}+4 \tau_{f}^{\prime} \gamma_{f}^{\prime}\left(2 N_{f}\right)^{b_{0}+c_{0}}
$$

where $\tau_{f}^{\prime}$ is the shear fatigue strength coefficient, $\gamma_{f}$ is the shear fatigue ductility coefficient, and $b_{0}$ and $c_{0}$ are another fatigue strength exponent and fatigue ductility exponent.

Jiang and Sehitoglu ${ }^{14)}$ proposed that the damage parameter should be defined as

$$
F P=\left\langle\sigma^{\max }\right\rangle \frac{\Delta \varepsilon}{2}+J \Delta \tau \Delta \gamma
$$

where < > denotes the MacCauley bracket, $\langle x\rangle=0.5(|x|+x) ; \sigma^{\max }$ is the maximum stress normal to the crack plane, $\Delta \varepsilon$ is the strain range normal to the crack plane, $\Delta \tau$ is the shear stress range on the crack plane and $\Delta \gamma$ is the shear strain range on the crack plane. The constant $J$, which is material and load-dependent, should be obtained from tension/torsion tests. The material plane with the largest $F P_{\max }$ fatigue parameter value is defined as the crack plane. The fatigue life to initiation is computed on this crack plane as

$$
\begin{aligned}
F P_{\max } & =\left(\left\langle\sigma^{\max }\right\rangle \frac{\Delta \varepsilon}{2}+J \Delta \tau \Delta \gamma\right)_{\max } \\
& =\frac{\left(\sigma_{f}^{\prime}\right)^{2}}{E}\left(2 N_{f}\right)^{2 b}+\sigma_{f}^{\prime} \varepsilon_{f}^{\prime}\left(2 N_{f}\right)^{b+c}
\end{aligned}
$$

(c) Ratchetting

The kinematic hardening, based on the non-linear Armstrong and Frederick law, has been modified by Jiang and Sehitoglu ${ }^{15)}$ to include several back-stress values of $X^{(i)}$

$$
\dot{X}^{(i)}=\sqrt{\frac{3}{2}} \dot{\lambda} c^{(i)}\left(r^{(i)} n_{\text {dev }}-\left\{\frac{\left|X^{(i)}\right|}{r^{(i)}}\right\}^{x^{(i)}} X^{(i)}\right), i=1,2, \ldots, M
$$

The exponent $\chi^{(i)}$ is given by

$$
\chi^{(i)}=\chi_{0}^{(i)}\left(2-n_{d e v}: \frac{X^{(i)}}{\left|X^{(i)}\right|}\right), i=1,2, \ldots, M
$$

Total back-stress $X$ is obtained by summation of all the back-stress values of $X^{(i)}$

$$
X=\sum_{i=1}^{M} X^{(i)}
$$

where $r^{(i)}, c^{(i)}$ and $\chi_{0}{ }^{(i)}(\mathrm{i}=1,2, \ldots, \mathrm{M})$ are material parameters obtained from experimental results.

Vincent et al. ${ }^{16)}$ introduced non-linear kinematic constitutive equations consistent with ratchetting modeling into the distortional model. They defined the active stresses $\hat{S}_{i}$ as the coordinates of the loading point in the moving system, which were:

$$
\hat{S}_{i}=Q_{i j}\left(S_{j}-X_{j}\right)
$$

where $S_{j}$ represents the coordinates of the loading point in the original coordinate system, $Q_{i j}$ is an orthogonal ten- sor that describes the rotation of the moving system, and $X_{j}$ is the translation vector of the moving system, which can be interpreted as a back-stress variable.

The yield surface was described by a parametric equation:

$$
\hat{S}_{i}=\left(d_{(i) k} t_{k}+\left[\left(d_{(i) k} t_{k}\right)^{2}-d_{(i) k} d_{(i) k}+R_{(i)}^{2}\right]^{1 / 2}\right) t_{i} \quad i=1,2
$$

where $d_{(i) k}$ denotes the distortional variables that can be considered as back-stress variables, $t_{k}$ represents the coordinates of the projecting radius vector, and $R_{(i)}$ is the size of the yield surface in the direction of uni-axial loadings and the other radius for the cross size of the yield surface. $R_{(i)}, X_{j}$ and $d_{(i) i}$ are developed as appropriate.

Lin et al. ${ }^{17)}$ proposed the elastic-visco-plastic damage model based on Chaboche and Rousselier's model. This is summarized as follows:

$$
\begin{aligned}
& \dot{\varepsilon}^{p}=\frac{3}{2}\left[\frac{J(\sigma-x) /(1-D)-k}{K}\right]^{n} \frac{\sigma^{\prime}-x^{\prime}}{J(\sigma-x)} \\
& \dot{x}_{1}=\frac{2}{3} c_{1}(1-D) \dot{\varepsilon}^{p}-\gamma_{1} x_{1} \dot{p} \\
& \dot{x}_{2}=\frac{2}{3} c_{2}(1-D) \dot{\varepsilon}^{p}-\gamma_{2} x_{2} \dot{p} \\
& x=x_{1}+x_{2} \\
& \sigma=E(1-D)\left(\varepsilon-\varepsilon^{p}-\varepsilon^{\theta}\right)
\end{aligned}
$$

where $\dot{p}$ is the effective plastic strain rate, and $J(\sigma-x)$ is the effective driving stress given by

$$
J(\sigma-x)=\left[\frac{3}{2}\left(\sigma^{\prime}-x^{\prime}\right):\left(\sigma^{\prime}-x^{\prime}\right)\right]^{1 / 2}
$$

The internal variable $x$ models the kinematic hardening that occurs in cyclic plasticity, while $D$ is a state variable representing combined material damage due to creep and cyclic plasticity. The total strain is given by $\varepsilon$ and the plastic and thermal strains by $\varepsilon^{\mathrm{p}}$ and $\varepsilon^{\theta}$ respectively. The material parameters $K, k, n, c_{1}, c_{2}, \gamma_{1}, \gamma_{2}$ and $E$ are determined by experiments. These material models can simulate ratchetting material response at a decaying ratchetting rate.

The model for predicting the time to fatigue crack initiation using ratchetting only was proposed by Kapoor 18). The number of cycles to crack initiation is calculated as

$$
N_{f}=\varepsilon_{c} / \Delta \varepsilon_{r}
$$

where $\varepsilon_{c}$ is a material constant determined by experiments, and $\Delta \varepsilon_{\mathrm{r}}$ is the equivalent ratchetting strain per cycle, calculated as

$$
\Delta \varepsilon_{r}=\sqrt{(\Delta \tilde{\varepsilon})^{2}+(\Delta \tilde{\gamma} / \sqrt{3})^{2}}
$$

where $\Delta \widetilde{\varepsilon}$ and $\Delta \widetilde{\gamma}$ are calculated as the incremental normal and shear strain acting on the crack plane per load cycle.

It is assumed that a piece of rail material in a railhead is subjected to cyclic constant amplitude loading 
from wheel loads, and that it will show one of the material responses mentioned above. One of these criteria will therefore be used to predict the crack initiation life corresponding to the material response elucidated through FEM analysis.

\subsection{Prediction of Crack Initiation Life for Squats}

Wheel/rail rolling contact is simulated in three-dimensional elastic-plastic problems using commercial multipurpose FEM code MARC. A longitudinal plane of symmetry is used in such a way that only one side of the track is modeled. Ringsberg ${ }^{2)}$ developed a strategy for life predictions of RCF crack initiation comprising elastic-plastic finite element analysis, multi-axial fatigue assessment of the life to fatigue crack initiation, and comparison of calculated results with verification tests or in-field observation. This research will refer to the procedure proposed by Ringsberg. The fatigue analysis assumes initially defect-free and homogeneous materials, i.e. a total-life approach is adopted. The capacity of a constitutive material model to simulate material responses as shown in Fig. 1 is important for the fatigue analysis. In this research, combined isotropic and kinematic hardening law is adopted to simulate material responses with a decaying hardening ratio and ratchetting ratio. Here, the initial hardening is assumed to be almost entirely isotropic, although after a certain amount of plastic strain the elastic range attains an essentially constant value, representing pure kinematic hardening. In order to provide the generality of this model, the fraction factor $f_{\mathrm{h}}$ is introduced:

$$
\begin{aligned}
& \bar{\sigma}_{\alpha}=\sigma_{y}+\left(1-f_{h}\right)\left(\bar{\sigma}-\sigma_{y}\right) \\
& \bar{\alpha}=f_{h}\left(\bar{\sigma}-\sigma_{y}\right)
\end{aligned}
$$

where $\bar{\sigma}_{\alpha}$ and $\bar{\alpha}$ represent hardening due to isotropic and kinematic contributions respectively, $\sigma_{\mathrm{y}}$ is the initial yield stress, $\bar{\sigma}$ is the von Mises stress and $f_{\mathrm{h}}$ is set at 0.5 .

Ringsberg ${ }^{1)}$ produced a rail FEM model representing $120 \mathrm{~mm}$ of track. The distribution of contact pressure and shear stress from wheel/rail contact were applied at the symmetry line on the top surface of the rail model, and were moved in the rolling direction. The normal contact road distributions were modeled according to the Hertz theory of rolling contact between two elastic non-conforming solids with a smooth and continuous contact surface. The tangential tractions were modeled as proportional to the normal contact loads via the friction coefficients. However, one disadvantage of this modeling is that the contact conditions are not updated when plastic deformation occurs during contact, and this may lead to a failure to account for any reduction in effective contact loads. Another drawback is that real contact between wheel and rail cannot be represented by Hertz theory. In this research, therefore, full contact conditions will be solved by employing constraints in the FEM simulation of the contact between wheel and rail. Namely, both wheel and rail are modeled using FEM mesh, and the wheel is then loaded and rotated in the rolling direction on the rail. The entire FEM model is indicated in Fig.2. The wheel and rail models are constructed using eight-node brick

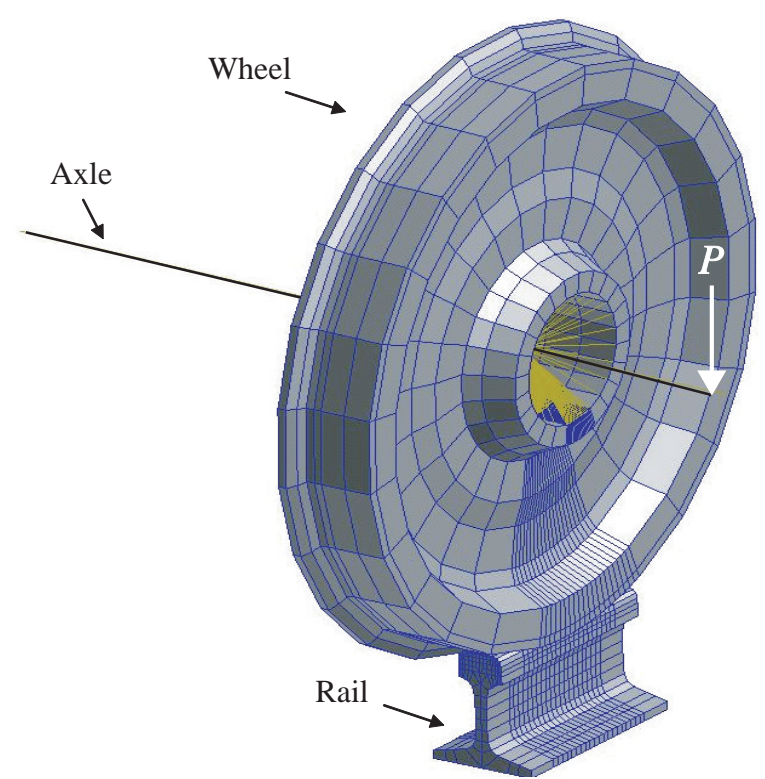

Fig. 2 FE model consisting of wheel, axle and rail

elements, and the number of elements in each model is 11,418 and 12,516 respectively. The wheel shape is that used for Shinkansen vehicles, and the diameter and configuration of the tread surface are $860 \mathrm{~mm}$ and conical respectively. The rail is modeled as a $60-\mathrm{kg}$ Shinkansen rail and represents $200 \mathrm{~mm}$ of track. Each FEM model is divided into an inner and an outer part. Wheel/rail contact occurs between these inner parts, and is quite essential for this analysis. These parts are therefore defined by the elastic-plastic material properties, and have larger mesh densities. In the case of the rail model, the width, length and depth of the inner part are $30 \mathrm{~mm}, 100 \mathrm{~mm}$ and $15 \mathrm{~mm}$ respectively. The outer parts represent the elastic surroundings, and the stress states here are far from severe. The volumes within these parts are therefore modeled using linear-elastic material. The difference in the element patterns of neighboring surfaces between the inner and outer parts is settled using multi-point constraint (MPC).

To rotate the wheel on the rail surface, the axle is modeled using elastic straight beam elements with a degree of freedom for rotation in three global directions. The beams have the same diameter and elastic modulus as the axle, and are connected to the wheel by rigid beam elements of a diameter fine enough $(0.1 \mathrm{~mm})$ to render their presence negligible. In reality, the wheel is pressfitted to the axle, but as the area of interest is the wheel/ rail contact region (in that the crack initiation lives for analysis are far from the wheel/axle fitted zone), differing connection methods seldom influence this area, and simplification can therefore be introduced. The axle is loaded at the node to which the vehicle weight is applied in reality with a concentrated force of $69 \mathrm{kN}$ (i.e. the wheel load) in the vertical direction, and is rotated by about 7 degrees in the rolling direction. This situation is considered as macroscopically pure rolling. The friction coefficient between the wheel and the rail is set at 0.3 .

According to this modeling, the contact conditions are updated when plastic deformation occurs in the contact 
Table 1 Mechanical properties of the wheel and rail steel

\begin{tabular}{|c|c|c|c|c|c|c|c|}
\hline $\begin{array}{c}E \\
(\mathrm{GPa})\end{array}$ & $v$ & $\begin{array}{c}\sigma_{y} \\
(\mathrm{MPa})\end{array}$ & $b$ & $c$ & $\varepsilon_{f}^{\prime}(\%)$ & $\begin{array}{c}\sigma_{f}^{\prime} \\
(\mathrm{MPa})\end{array}$ & $J$ \\
\hline 209.82 & 0.29 & 472 & -0.089 & -0.559 & 10.3 & 936 & 0.2 \\
\hline
\end{tabular}

area, and a reduction in effective contact loads can be taken into consideration. The rail is inclined on the inside by 1.43 degrees to the vertical direction, and all nodes on the bottom surface are constrained to only that direction. Furthermore, two distinct constraints are introduced to the cross-sections of the rail model; the first is that all degrees of freedom of the nodes at both cross-sections are fixed (model I). The second is that all degrees of freedom of the nodes are fixed at one cross-section, while those at the other cross-section are free (model II). The actual situations are considered to fall somewhere between these constraints. The node of the axle model on the longitudinal plane of symmetry is fixed in the horizontal direction. The mechanical properties of the steel in the wheels and rails used for this FEM analysis are given in Table 1.

\section{Results}

Ten wheel passages were performed for models I and II, and the crack plane angle and number of cycles to crack initiation were then estimated following one of the criteria outlined in 2.2. Each passage was divided into four phases i.e. (1) the wheel is on the rail surface and the wheel load is applied to the axle; (2) the wheel is rotated on the rail surface keeping the load constant; (3) after the wheel is rotated to the pre-defined angle, the load is removed and the wheel is separated from the rail surface; (4) the wheel is returned to the initial position.

The example of von Mises stress in the railhead for model II during the tenth wheel passage is shown in Fig.3. This figure indicates the vicinity of the contact region and just the middle of the rail's longitudinal direction. The stress-strain curves near the contact surface of the rail model after ten wheel passages showed a plastic shakedown material response. Among the criteria for such responses, the one proposed by Jiang and Sehitoglu ${ }^{14)}$ seemed to hold up under RCF conditions in comparison and evaluation. The criterion proposed by Jiang and Sehitoglu is therefore used to predict the position and direction of cracks and the fatigue life. The criterion was applied to all elements in all rail cross-sections for every load step in the inner part of the rail model. The crack plane (i.e. the critical plane) for crack initiation was calculated using the results of FEM simulation. This plane is defined as the material plane that accumulates the largest amount of damage $F P_{\max }$ according to Eq.(7) during each wheel passage. The angles that define the direction of the crack plane are shown in Fig.4, where $v$ denotes the rolling direction of the wheel. Angle $\theta$ is the angle between the $x$-axis and the crack plane, and $\varphi$ is the angle between the normal of the crack plane and the $x$-axis as seen from above. These are referred to by Ringsberg et al. ${ }^{1)}$.

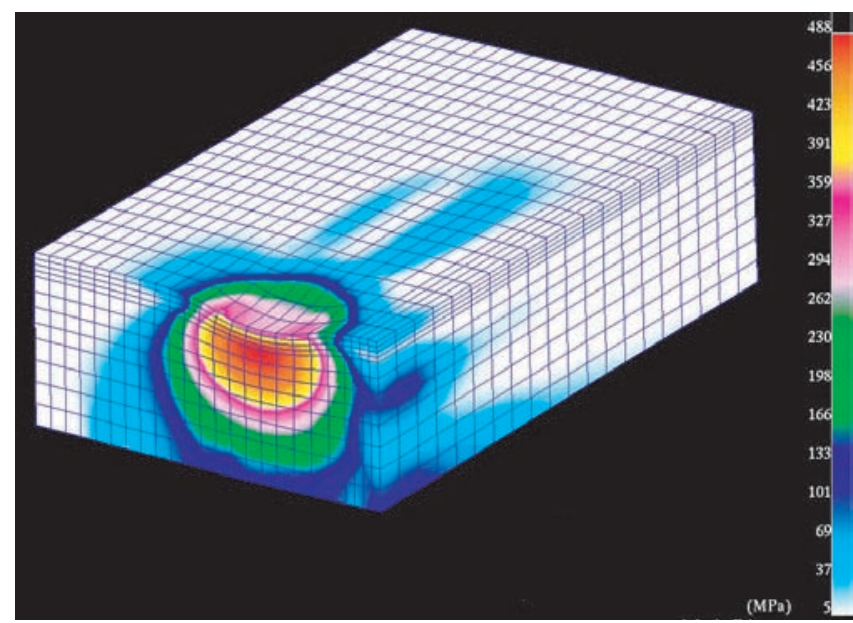

Fig. 3 von Mises stress distribution during tenth wheel passage

(Model II ; magnification factor for deformation is 50 )

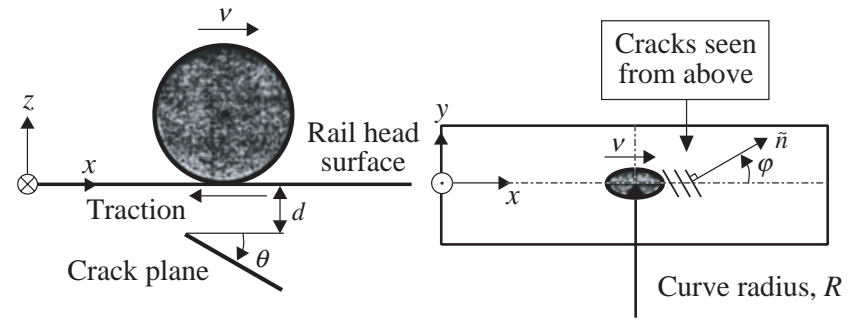

Fig. 4 Definition of angles for the crack plane for crack initiation $(v$ denotes the rolling direction of the running vehicle) ${ }^{2)}$.

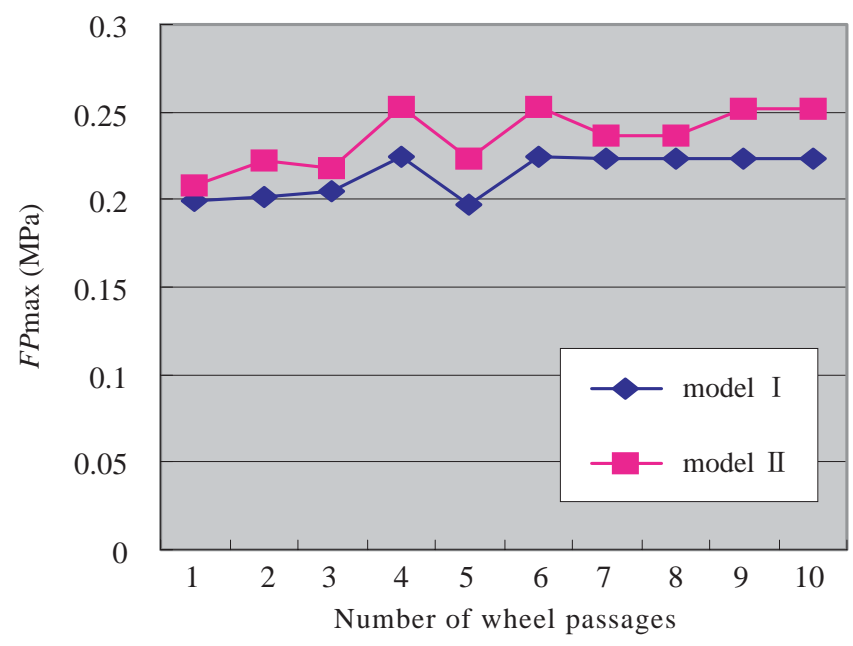

Fig. 5 Variations of $\boldsymbol{F P}_{\max }$ according to the number of wheel passages.

The crack initiation life was calculated using Eq.(8) for $F P_{\text {max }}$. The variations of $F P_{\max }$ caused by wheel passage are indicated in Fig.5. After ten wheel passages, $F P_{\max }$ is seen to almost converge to 0.22 for model I, and to 0.25 for model II. Table 2 summarizes the angles of the crack planes, the $F P_{\max }$ maximum damage parameter values and the crack initiation cycles calculated after ten wheel passages for model I and model II respectively. 
Table 2 Predicted results for crack angle, $\boldsymbol{F P}_{\max }$ and crack initiation lives

\begin{tabular}{|c|c|c|c|c|}
\hline Model & $\theta(\mathrm{deg})$ & $\varphi(\mathrm{deg})$ & $F P_{\max }(\mathrm{MPa})$ & $N_{f}$ \\
\hline Model I & 0 & 0 & 0.223 & $7.46 \times 10^{6}$ \\
\hline Model II & 25 & 10 & 0.252 & $3.86 \times 10^{6}$ \\
\hline
\end{tabular}

\section{Discussion}

The FEM model for the analysis of RCF cracks in wheel/rail systems developed here is the improved version proposed by Ringsberg ${ }^{1)}$. In this research, both wheel and rail are modeled using FEM mesh, and the wheel is loaded and rotated in the rolling direction on the rail. According to this modeling, the contact conditions are updated when plastic deformation occurs in the contact area, and a reduction in effective contact loads can be taken into consideration. However, the computation is very time-consuming, with FEM analysis for just one wheel passage taking about 44 hours using ORIGIN 3000 (1.2Gflps per CPU). Case studies can be conducted by varying, for example, the material parameters, the geometry of the wheel and the rail and the loading conditions without difficulty. This research targets squats in the railhead, although shelling in the wheel tread can also be dealt with using the same procedure as seen in this model.

Ringsberg ${ }^{1)}$ performed numerical prediction of the initiation life for cracks generated on the top of the railhead. As outlined in 2.4, however, his strategy relies on the distribution of contact pressure and shear stresses from the wheel/rail contact being applied at the symmetry line on the top surface of the rail model and moved in the rolling direction. The wheel load was $59 \mathrm{kN}$, and the friction coefficients in the longitudinal and transverse directions were 0.25 and 0.31 respectively. The mechanical properties of the rail steel were about the same, except the yield stress was 400.1 MPa in Ringsberg's model. FEM simulation involved 16 wheel passages, after which the results were evaluated in fatigue analysis. Under these conditions, the wheel/rail contact region showed plastic shakedown material response, and the number of wheel passages to crack initiation was predicted as $6.6 \times$ $10^{6}$. Unfortunately, the calculated angle of the crack plane was not shown in the literature. Considering that the values for the mechanical properties of the materials for fatigue and maximum contact pressure are not strictly uniform between this study and Ringsberg's numerical conditions, the agreement is considered to be satisfactory.

Limited experimental data is found in literature to verify the numerical results in this research. Li et al. ${ }^{19)}$ observed short-crack behavior in the rolling contact area of medium carbon steel rollers $(0.43 \% \mathrm{C}, 0.42 \% \mathrm{Si}, 0.52$ $\% \mathrm{Mn}, 0.026 \% \mathrm{~S}, 0.023 \% \mathrm{P})$ under the conditions of pure rolling and several values of contact pressure. Fatigue properties such as $\sigma_{f}^{\prime}, \varepsilon_{f}^{\prime}, b$ and $c$ are not clear, but these values can be considered similar to those of the current research according to available literature ${ }^{20)}$. The initiation and propagation of short cracks were traced through microscopic observation, and based on this, the initiation life and surface distress life of the RCF of the roller was measured. The tests were performed on a two-disc wear machine, and both specimen diameters were $60 \mathrm{~mm}$ under maximum contact pressures ranging from 580 to 1360 $\mathrm{MPa}$ at a zero slide/roll ratio and a rotation of 400 rounds/ min. The curves of crack length $a$ vs. the number of cycles $N$ under different contact pressures obtained from these tests are shown in Fig.6. The maximum pressure generated in the wheel/rail contact region in the current research was calculated at about $1092 \mathrm{MPa}$ if the Hertz theory is applied. When the curve of $P_{0}=1100 \mathrm{MPa}$ in Fig.6 was extrapolated along the last slope to a crack length of $0.5 \mathrm{~mm}$, (i.e. the crack length at which initiation is defined as having occurred in the current research), the corresponding number of cycles was about $2 \times 10^{6}$. The number of cycles for the initiation of $0.5 \mathrm{~mm}$-length cracks using numerical prediction in the current research is $7.46 \times 10^{6}$ for model I and $3.86 \times 10^{6}$ for model II. The cracks propagate along the circumferential direction of the roller at the final stage in the rolling contact tests carried out by Liu et al., representing $\theta=0^{\circ}$ for the crack plane direction in the current research. The angle predicted using a numerical procedure in the current research is $\theta=0^{\circ}$ for model I and $\theta=25^{\circ}$ for model II. Unfortunately, there are no results for $\varphi$ in Liu's paper. The mechanical properties of the materials for fatigue and maximum contact pressure are not strictly the same between the current numerical and laboratory test conditions, and the geometry and scale of the wheel and rail and the specimens are of course different. The effect of scale should therefore be considered, and the agreement may be satisfactory.

From field observations of the Tokaido Shinkansen track, it was reported that crack initiation was seen at intervals of several hundred meters along the rail's longitudinal direction after about $2 \times 10^{7}$ wheel passages under a combination of an average wheel load of $80 \mathrm{kN}$, a wheel radius of $910 \mathrm{~mm}$ and a $60-\mathrm{kg}$ rail ${ }^{21)}$. Rewriting

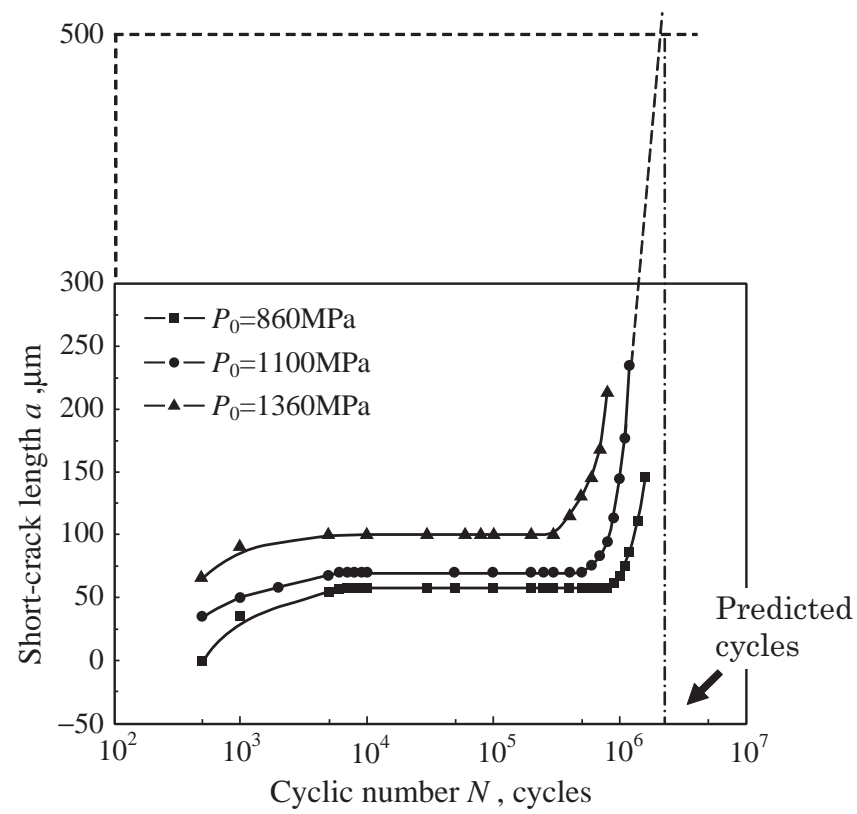

Fig. 6 Curves of short-crack length $a$ vs. the number of cycles $N$ with different maximum contact pressure $P_{0}{ }^{19)}$. The dotted lines give the cyclic number of crack propagation to $0.5 \mathrm{~mm}$ by extrapolation. 
once again, the analytical results of the current research showed earlier initiation at $3.86 \times 10^{6}$ and $7.46 \times 10^{6}$. In the numerical simulation of the current research, it was assumed that the contact positions were exactly the same. In actual wheel/rail contact situations, however, the values vary with each wheel passage, and the accumulated damage is therefore considered to be less. In addition, the crack size at which initiation was defined to have occurred is $0.5 \mathrm{~mm}$ in the current research. However, the crack length must be larger to be seen by the naked eye on actual rail surfaces, and in this respect also, the predicted results must be earlier than the field data.

This paper shows an example of applying the finite element model for the analysis of rolling contact fatigue cracks in wheel/rail systems for squats on the railhead. However, the model can also be applied to shelling in the wheel tread. The application of this method is expected to enable accurate estimation of wheel integrity in the future.

\section{Conclusions}

In this study, a numerical model that can be used to estimate the fatigue crack initiation of squats on the railhead due to RCF was developed. Both wheel and rail were modeled using FEM mesh, and the wheel was loaded and rotated in the rolling direction on the rail. The crack initiation lives were estimated using the Jiang and Sehitoglu criterion for plastic shakedown material responses. The numerical results were compared with other numerical analysis, laboratory test data and field observations. These results showed a reasonable correlation between the number of wheel passages to crack initiation and the direction of cracks by considering that many sources were not taken into account in the current analysis, and the differences were discussed in detail.

\section{References}

1) Ringsberg J. W. et al., "Rolling contact fatigue of railsfinite element modelling of residual stresses, strains and crack initiation," Proceedings of the Institution of Mechanical Engineers Part F, Vol.214, pp.7-19, 2000.

2) Ringsberg J. W., "Life prediction of rolling contact fatigue crack initiation," International Journal of $\mathrm{Fa}$ tigue, Vol.23, pp.575-586,2001.

3) Bogdański S., Brown M. W., "Modeling the three-dimensional behavior of shallow rolling contact fatigue cracks in rails," Wear, Vol.253, pp.17-25, 2002.

4) Dubourg M. C., Lamacq V., "A predictive rolling contact fatigue crack growth model: onset of branching, direction, and growth - role of dry and lubricated conditions on crack patterns," ASME Journal of Tribology, Vol.124, pp.680-688, 2002.

5) Suresh, S., Fatigue of Materials, Cambridge Univer- sity Press, 1998.

6) Johnson, K. L., "The strength of surfaces in rolling contact," Proceedings of the Institution of Mechanical Engineers Part E, Vol.203, pp.151-163, 1989.

7) Sines G., "Failure of materials under combined repeated stresses with superimposed static stresses," Technical note 3495, Nat. Advisory Council for Aeronautics, Washington, DC. 1955.

8) Crossland, G., "Effect of large hydrostatic pressures on the torsional fatigue strength of an alloy steel," Proceedings of the International Conference of Fatigue of Metals, I. MechE., London, pp.138-149, 1956.

9) Dang Van, K. et al., "Criterion for high cycle fatigue failure under multiaxial loading," Biaxial and Multiaxial Fatigue (edited by Brown, M. and Miller,K.J.), Mechanical Engineering Publications, London, pp.459-478, 1989.

10) Smith, K. N., "A stress-strain function for the fatigue of metals," Journal of Materials JMLSA, Vol.5, pp.767778, 1976.

11) Socie, D. F., "Multiaxial fatigue damage models," Journal of Engineering Materials and Technology, Vol.109, pp.292-298, 1987.

12) Rolovic, R. and Tipton, S. M., "An energy based critical plane approach to multiaxial fatigue analysis," Fatigue and Fracture Mechanics, ASTM STP 1332, West Conshohocken, PA, pp.599-613, 1999.

13) Chen, X. et al., "A critical plane-strain energy density criterion for multiaxial low-cycle fatigue life under non-proportional loading," Fatigue of Engineering Materials and Structures, Vol.22, pp.679-686, 1999.

14) Jiang, Y. and Sehitoglu, H., "A model for rolling contact failure," Wear, Vol.224, pp.34-49, 2000.

15) Jiang, Y. and Sehitoglu, H., "Rolling contact stress analysis with the application of a new plasticity model," Wear, Vol.191, pp.35-44, 1996.

16) Vincent, L. et al., "An improvement of multiaxial ratchetting modeling via yield surface distortion," Transactions of the ASME Journal of Engineering Materials and Technology, Vol.124, pp.402-411, 2002.

17) Lin, J. et al., "Approximate method for the analysis of components undergoing ratchetting and failure," Journal of Strain Analysis, Vol.33, No.1, pp.55-65, 1998.

18) Kapoor, A., "A re-evaluation of the life to rupture of ductile metals by cyclic plastic strain," Fatigue and Fracture of Engineering Materials and Structures, Vol.17, pp.201-219, 1994.

19) $\mathrm{Li}$, Y. et al., "Vertical short-crack behavior and its application in rolling contact fatigue," International Journal of Fatigue, Vol.28, pp.804-811, 2006.

20) National Research Institute for Metals, NRIM Fatigue Data Sheet, No.2, Data Sheets on Fatigue Properties of S35C (0.35C) Steel for Machine Structural Use, 1978.

21) Sugiyama, T. and Matsuyama, S., "Contact fatigue failures of rail - shelling - (in Japanese)," Junkatu, Vol.30, No.6, pp.393-397, 1985. 